Lymphology 53 (2020) 81-87

\title{
THORACIC DUCT STENT-GRAFT DECOMPRESSION WITH 3-MONTH PATENCY: REVISITING A HISTORICAL TREATMIENT OPTION FOR PORTAL HYPERTENSION
}

\author{
H. McGregor, G. Woodhead, M. Patel, C. Hennemeyer
}

Department of Medical Imaging, University of Arizona, Tucson, Arizona USA

\section{ABSTRACT}

This report introduces the rationale for thoracic duct stent-graft decompression in cirrhotic patients with portal hypertension and provides a case example with 3-month stentgraft patency. Thoracic duct flow and pressure are elevated in cirrhosis. Historically, complications of portal hypertension have been successfully treated with external drainage of the thoracic duct or surgical lymphovenous bypass. A 45-year-old woman with cirrhosis, chronic portosplenomesenteric thrombosis, and acute variceal hemorrhage underwent percutaneous thoracic duct stent-graft placement across the lymphovenous junction.

The hemorrhage subsequently resolved and follow up endoscopy demonstrated decompression of the bleeding varices. Venography 40 days later demonstrated a partially patent stent-graft with fibrin sheath formation distally. The stent-graft was extended distally to the right atrium and was fully patent on venography 3 months later. The patient had no further episodes of hemorrhage.

Keywords: stent-graft, thoracic duct, decompression, cirrhosis, portal hypertension

The advent of modern angiographic techniques and equipment has renewed interest in lymphatic interventions. Of potential interest to Interventionalists is the relationship between the lymphatic and portal systems. Thoracic duct lymph flow and pressure are elevated in cirrhosis and portal hypertension (1). In 1969 Witte et al demonstrated a mean thoracic duct lymph flow rate of 6.8 milliliters per minute $(\mathrm{mL} / \mathrm{min})$ in 41 cirrhotic patients compared to $0.82 \mathrm{~mL} /$ min in 43 controls. Mean thoracic duct pressure was 20.6 millimeters of Mercury $(\mathrm{mmHg})$ in the cirrhotic patients and 8.5 $\mathrm{mmHg}$ in controls. Eleven patients in this study with cirrhosis subsequently underwent surgical portosystemic shunt creation. In these patients, mean thoracic duct lymph flow rates decreased to $1.8 \mathrm{~mL} / \mathrm{min}$ and thoracic duct pressure decreased to $14.7 \mathrm{mmHg}$ after portal decompression (1). In 1962, Dumont and Mulholland provided evidence supporting the clinical relevance of increased lymphatic flow and pressure in cirrhosis, presenting positive clinical responses in the management of portal hypertension (improvement in ascites or cessation of variceal hemorrhage) after external thoracic duct drainage in 8 patients. In addition, portal pressure declined by $25 \%$ 20 minutes after commencing external drainage (2). Ongoing fluid and nutritional losses and immunosuppression prevents the use of external thoracic duct drainage as a long-term treatment option (3).

The thoracic duct drains into the central venous system at the lymphovenous junction, where valves act to prevent reflux of venous 
blood in normal physiologic conditions (4). Failure of the lymphovenous junction to adequately accommodate increased lymph flow may contribute to elevated thoracic duct pressures and lymphatic hypertension in high lymph flow states such as cirrhosis. The thoracic duct is abnormally dilated in cirrhosis, measuring at least 2-3 times larger than in normal patients, but the lymphovenous junction does not change in size (5). In several animal models, bypassing the lymphovenous junction resulted in an increase in thoracic duct flow $(6,7)$. The hypothesis that the lymphovenous junction is an area of relative obstruction to lymph flow in cirrhosis led investigators to explore lymphovenous bypass as a potential treatment option. In 1974, Donini et al performed thoracic duct to internal jugular vein anastomoses in $\mathbf{1 5}$ patients with cirrhosis and refractory ascites. Ascites completely resolved in $\mathbf{1 0}$ patients and portal pressure decreased from $20.8 \mathrm{mmHg}$ to $14.6 \mathrm{mmHg}$ in the 4 patients in whom splenic pulp pressure was measured (8).

A recent article has demonstrated the technical feasibility of reconstructing the lymphovenous junction with a stent-graft after iatrogenic injury (9). However, patency data in this case was not available. This report builds on that technique and describes relief of variceal hemorrhage associated with cirrhosis and portal hypertension after stent-graft deployment across the lymphovenous junction. Direct evidence of 3-month stent-graft patency is also provided.

\section{Thoracic Duct Decompression}

Institutional Review Board approval was not required for this case report. A 45-year-old woman with a history of hepatitis $\mathrm{C}$ virus and alcohol induced cirrhosis complicated by chronic portosplenomesenteric venous thrombosis and recurrent upper gastrointestinal hemorrhage presented to the Emergency Department with large volume hematemesis. She underwent emergent esophagogastroduodenoscopy (EGD), which demonstrated type 2 gastroesophageal varices with evidence for active hemorrhage. The active hemorrhage prevented adequate visualization for endoscopic intervention and a Minnesota tube was placed. The Interventional Radiology service was consulted for further management. Given the chronic complete portosplenomesenteric venous thrombosis, portal vein recanalization with transjugular intrahepatic portosystemic shunt (TIPS) was not feasible and the decision was made to proceed with lymphangiography and possible thoracic duct stent-graft decompression.

Intranodal lymphangiography was performed with injection of $6 \mathrm{ml}$ of ethiodized oil (Lipiodol, Guerbet, Villepinte, France) through 25-gauge Chiba needles (Cook Medical, Bloomington, IN, USA) into right and left inguinal lymph nodes. The cisterna chyli was visualized 42 minutes after commencement of the procedure. The thoracic duct was completely opacified 65 minutes after injection. The thoracic duct was dilated, measuring 13 $\mathrm{mm}$, and contained several well-visualized valves (Fig. 1). A 22-gauge Chiba needle (Cook Medical, Bloomington, IN, USA) was advanced percutaneously from immediately superior to the sternal notch into the thoracic duct, proximal to its apex. An 0.014" microwire (Synchro, Stryker, Kalamazoo, MI, USA) was then advanced through the needle and coiled in the thoracic duct (Fig. 2). A microcatheter (Echelon 14, Medtronic, Dublin, Ireland) was advanced over the wire into the thoracic duct and thoracic ductography was performed with iodinated contrast (Isovue 300, Bracco, Milan, Italy) to opacify the lymphovenous junction. This demonstrated multiple dilated lymphatic channels surrounding the lymphovenous junction (Fig. 3). The microwire was reintroduced and used to navigate through the lymphovenous junction into the superior vena cava. The right femoral vein was then accessed with an $8 \mathrm{~F}$ sheath (Pinnacle Destination, Terumo, Tokyo, Japan) and the microwire was captured and retracted through the femoral vein sheath using a snare (Atrieve, Argon Medical, Frisco, TX, USA). A $4 \mathrm{~mm} \mathrm{x} 4 \mathrm{~cm}$ 


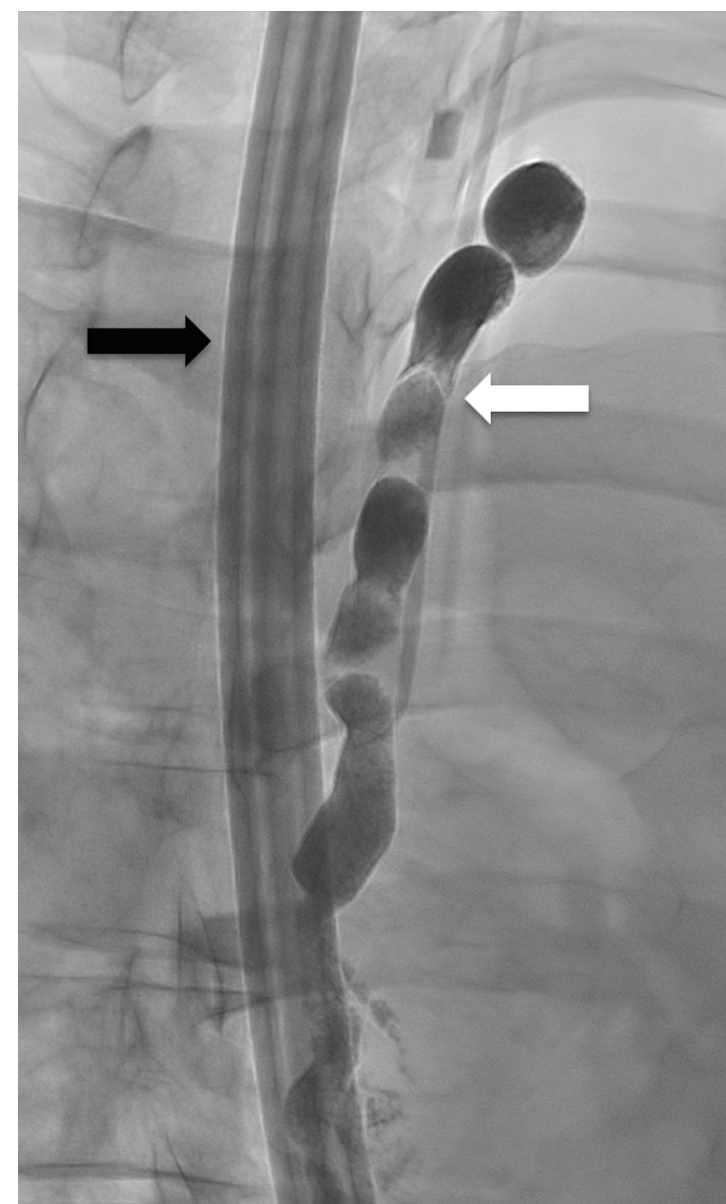

Fig. 1. Lymphangiographic image after the intranodal injection of ethiodized oil demonstrating thoracic duct dilation and tortuosity. Note the presence of a valve within the thoracic duct (white arrow) and Minnesota tube (black arrow).

balloon catheter (Pacific Plus, Medtronic, Dublin, Ireland) was then advanced over the microwire through the femoral sheath and inflated across the lymphovenous junction (Fig. 4). A $5 \mathrm{~mm}$ by $10 \mathrm{~cm}$ stent-graft (Viabahn, Gore, Newark, DE, USA) was advanced over the wire and deployed across the lymphovenous junction, with the proximal end positioned at the apex of the thoracic duct and the distal end proximal to the confluence of the brachiocephalic veins. The guidewires and sheaths were then removed and hemostasis achieved with manual

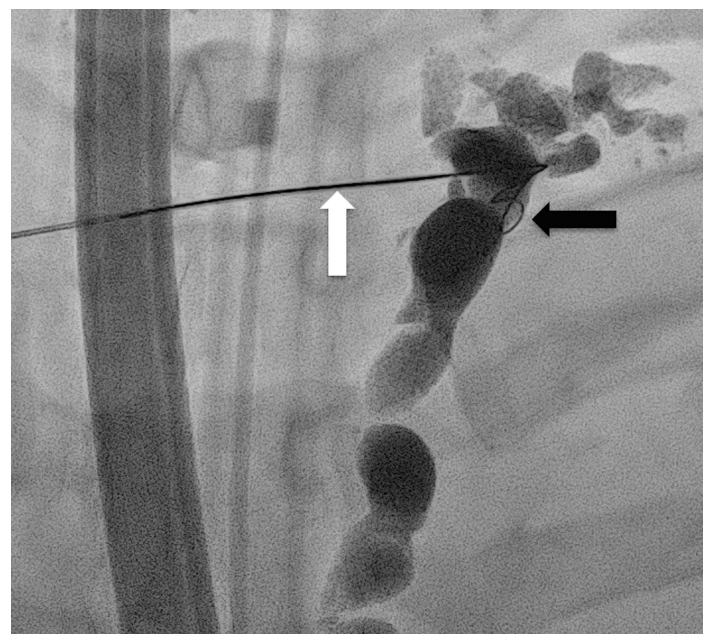

Fig. 2. Fluoroscopic image depicting percutaneous access into the thoracic duct. Note the 22-gauge needle inserted just superior to the sternal notch (white arrow) and wire coiled in the thoracic duct (black arrow).

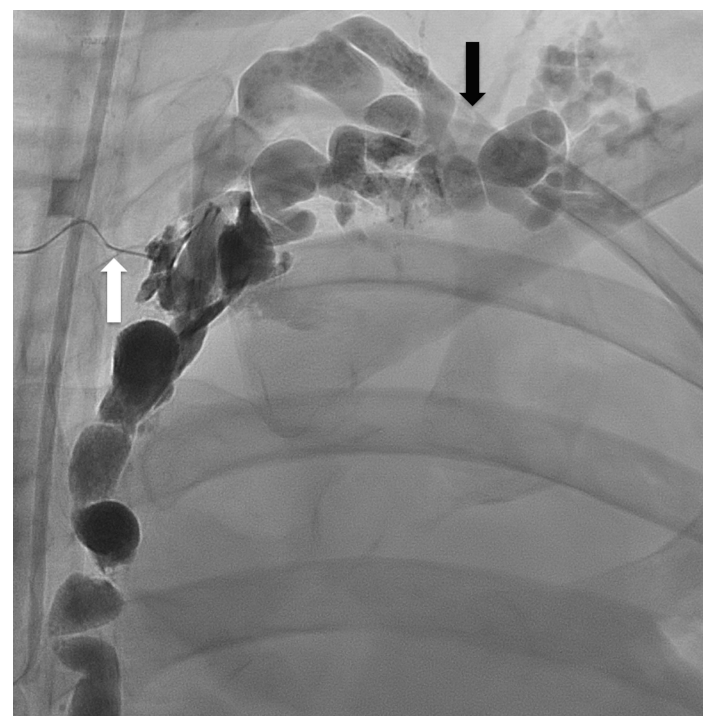

Fig. 3. Fluoroscopic image after instillation of contrast through the microcatheter (white arrow) depicting dilated lymph ducts (black arrow) surrounding the lymphovenous junction.

compression. Spontaneous flow of ethiodized oil was noted through the stent-graft (Fig. 5).

The patient was hemodynamically stable after the procedure and the Minnesota tube 


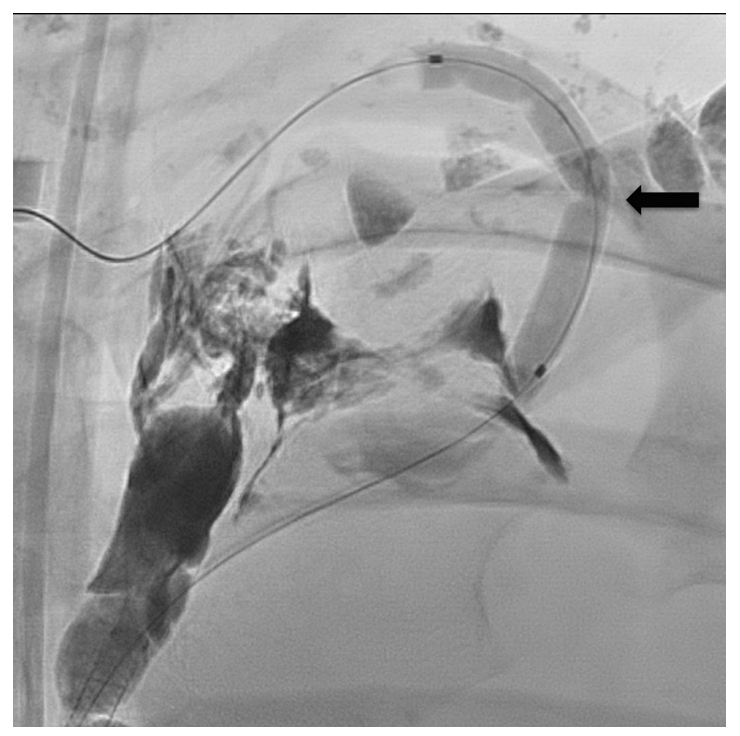

Fig. 4. Fluoroscopic image demonstrating balloon dilation of the lymphovenous junction. Note the waist on the balloon (black arrow).

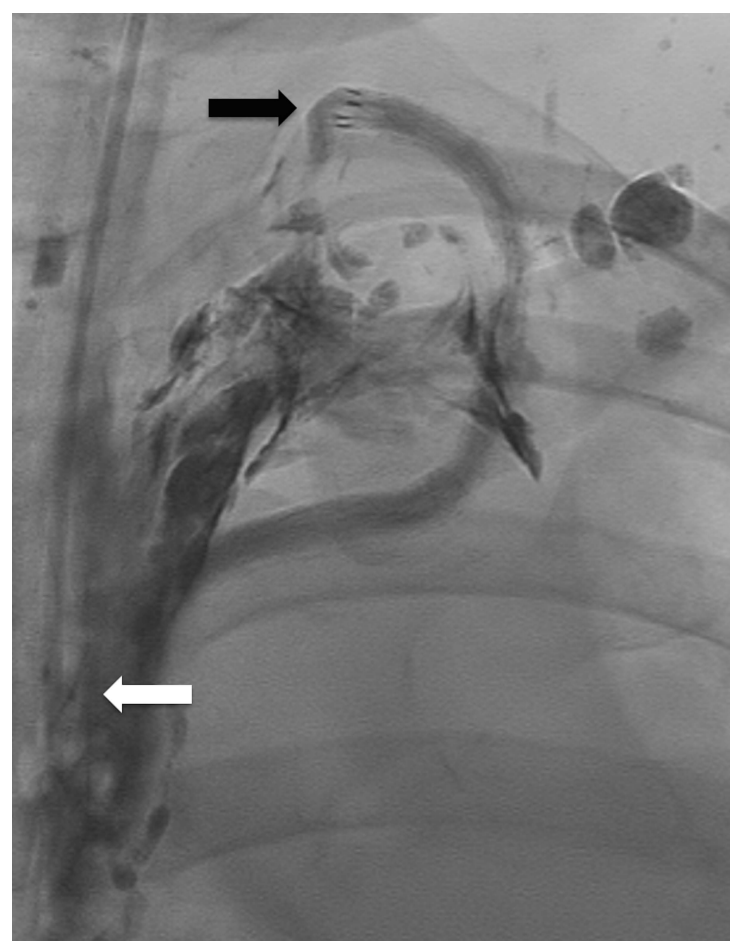

Fig. 5. Fluoroscopic image after stent-graft deployment demonstrating spontaneous flow of ethiodized oil into the central venous system. Note the location of the proximal (black arrow) and distal (white arrow) ends of the stent-graft. was removed on post procedure day 3. A repeat EGD was then performed and demonstrated decompression of the previously noted type 2 varices. On post procedure day 9 , pedal lymphoscintigraphy was performed to assess stent-graft patency. Two millicuries of Technetium $99 \mathrm{~m}$ radiolabeled sulfur colloid (Tc99-SC) were injected intradermally into the web spaces of each foot. Planar and single photon emission computed tomography (SPECT) images were acquired at 35, 44, 65, and 198 minutes after injection. These images demonstrated signal accumulation within the liver 44 minutes after the injection, indicating rapid transit of lymph through the thoracic duct and patency of the stent-graft. Additionally, a focal collection of radiotracer was localized immediately lateral to the stent-graft in the supra-clavicular region, likely reflecting stasis of lymph in a dilated lymphatic channel whose outflow had been occluded by the adjacent stent-graft (Fig. 6). The patient was discharged on post procedure day 10 .

Thirty days after discharge, the patient represented to the Emergency Department with lower extremity swelling and large volume hematemesis. Following resuscitation, the patient was brought immediately to the Interventional Radiology suite for venography and assessment of the thoracic duct stent-graft. Femoral venous access was obtained and unsuccessful attempts were made to catheterize the stent-graft in retrograde fashion. The stent-graft was then percutaneously accessed via a direct stick with a 21-gauge needle. An 0.018 " wire was then advanced antegrade through the stentgraft and into the subclavian vein. A 4 French sheath was advanced over the wire and angiography was performed. This demonstrated a patent stent-graft with sluggish antegrade flow and significant retrograde flow into dilated lymphatics proximal to the lymphovenous junction (Fig. 7). A focal filling defect with an associated linear area of contrast staining was noted at the distal aspect of the stent- 


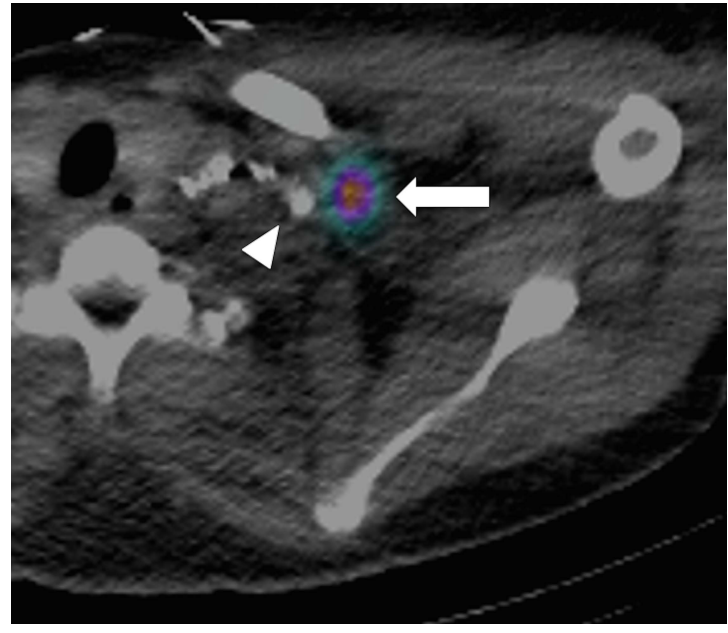

Fig. 6. SPECT/CT fusion image demonstrating radiolabeled sulfur colloid accumulation (arrow) lateral to the stent-graft (arrowhead), likely within a dilated lymphatic.

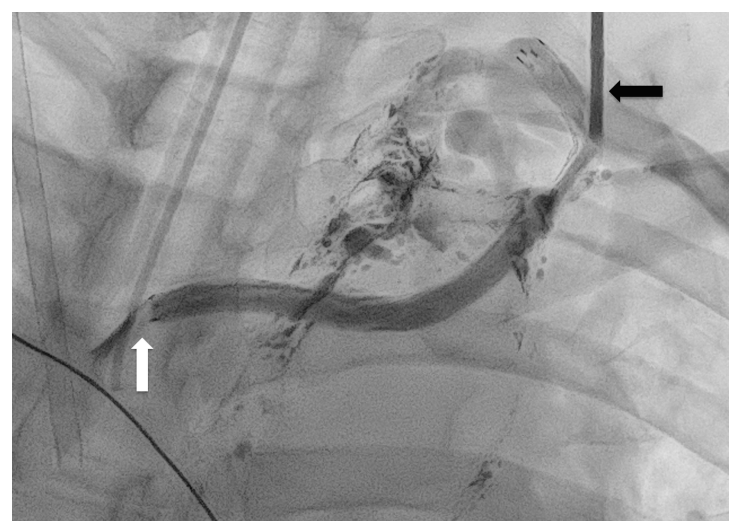

Fig. 7. Angiographic image 40 days after initial stent-graft placement demonstrating a patent stentgraft with a fibrin sheath distally (white arrow) Note the 4 French sheath within the stent-graft (black arrow).

graft, compatible with a fibrin sheath. Pressure within the stent-graft was $19 \mathrm{mmHg}$, compared to a central venous pressure of 8 $\mathrm{mmHg}$. An $0.035 "$ guidewire was then advanced through the sheath to the inferior vena cava. This was captured by a snare advanced from a femoral sheath and through and through wire access was established as previously described. A $5 \mathrm{~mm}$ by $4 \mathrm{~cm}$ balloon catheter (Conquest, Bard, Murray Hill, NJ, USA) was advanced over the wire and inflated across the distal aspect of the stentgraft. Repeat angiography demonstrated improvement in flow with some remaining contrast-stained material adherent to the distal aspect of the stent-graft. A snare (Atrieve) was used to extract a $3 \mathrm{~mm}$ by $2 \mathrm{~mm}$ piece of tan colored tissue, which was sent for pathological assessment. Repeat pressures within the stent-graft measured $9 \mathrm{mmHg}$, decreased from $19 \mathrm{mmHg}$. The stent-graft was then extended proximally to the ascending thoracic duct and distally to the right atrium by deploying 2 additional $5 \mathrm{~mm}$ x $10 \mathrm{~cm}$ stent-grafts (Viabahn). Histologic examination of the specimen revealed extensive fibrin formation. The patient stabilized and EGD on post-procedure 2 days demonstrated continued decompression of the previously described type 2 gastroesophageal varices and isolated esophageal varices, which were banded. The lower extremity swelling also resolved. The patient was subsequently extubated and was discharged in stable condition on post procedure day 6 . The patient presented for surveillance angiography 3 months after discharge, which demonstrated a patent stent-graft (Fig. 8). No further episodes of hemorrhage occurred during the follow-up period.

\section{DISCUSSION}

This report serves 2 purposes: 1) To conceptualize and introduce a potential novel treatment option for complications related to portal hypertension in cirrhosis and 2) To provide data for patency of thoracic duct stent-grafts.

Research into lymphatic interventions in cirrhosis slowed after the promising successes of the 1960s and 1970s, possibly due to the advent of liver transplantation, development of the TIPS, and advances in endoscopic interventions. There does however exist a patient population that suffers from complications of cirrhosis and portal 


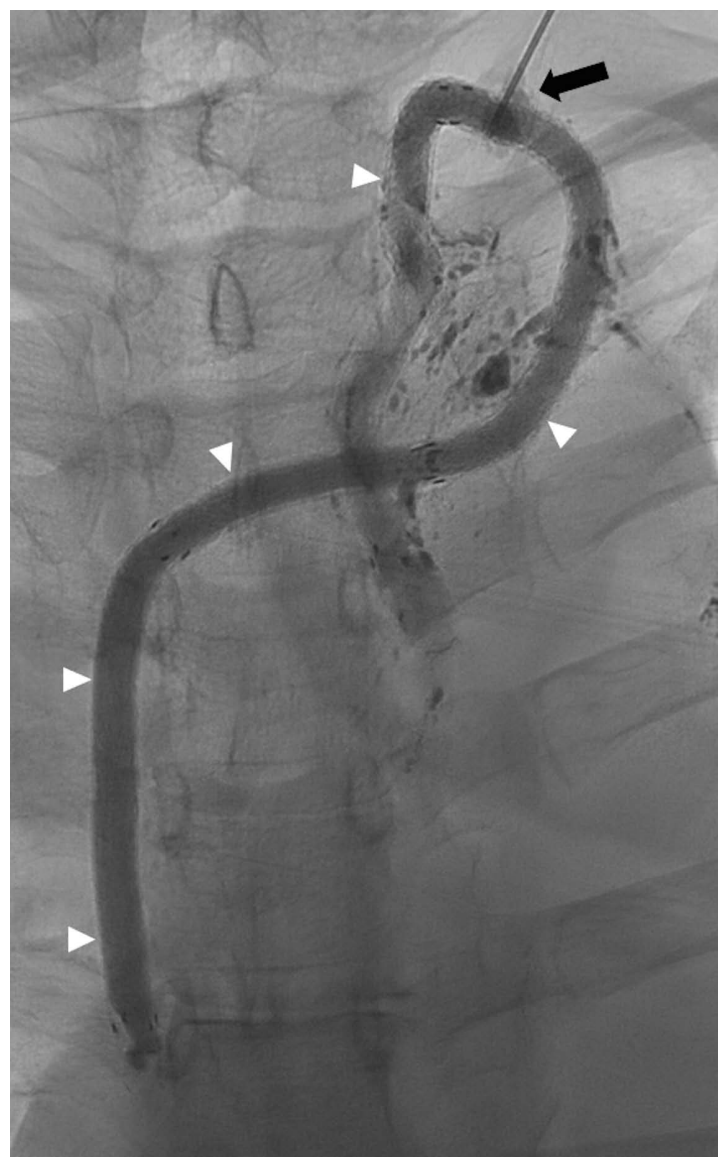

Fig. 8. Angiographic image 3-months after stentgraft extension. Contrast was injected through a 21 gauge needle (black arrow), demonstrating a patent stent-graft which extends from the ascending thoracic duct to the right atrium (arrowheads).

hypertension who are not TIPS candidates and do not yet qualify for liver transplantation. A procedure to relieve symptoms in these patients, whether variceal bleeding or refractory ascites, could improve quality of life and prevent mortality. The previously discussed evidence for the utility of thoracic duct decompression in cirrhosis by bypassing or opening the relative obstruction at the lymphovenous junction is promising and further studies should be pursued with the use of modern interventional tools. In the current case, the patient's variceal hemorrhage resolved immediately after stentgraft placement. Interestingly, repeat hemorrhage and leg swelling occurred when flow through the stent-graft was compromised by a fibrin sheath, resulting in elevated thoracic duct pressure $(19 \mathrm{mmHg})$. Intermittent and recurrent variceal hemorrhage may occur regardless of intervention, but the correlation between stentgraft malfunction and rebleeding in this case supports the concept of relieving portal hypertension by thoracic duct decompression.

Thoracic duct stent-grafting has previously been described for chyle leaks but no patency data have been published to date $(9,10)$. Good clinical success has been achieved with stentgrafting, but the lack of patency data has led some researchers to hypothesize that these devices may occlude shortly after placement and act as embolization material. The case described in this article provides much needed evidence for patency. Lymphoscintigraphy demonstrated indirect evidence for patency of the stent-graft 9 days after the procedure. Direct angiographic interrogation 40 days after placement demonstrated a partially patent stent-graft with findings consistent with fibrin sheath formation, similar to what is seen in malfunctioning port catheters. This finding was confirmed by the presence of fibrin on a retrieved specimen. After distal extension to the right atrium, the stent-graft was fully patent on direct angiographic interrogation at 3 months. Several factors may have contributed to the patency in this case. Firstly, the accelerated lymph production and flow in cirrhosis may limit reflux of venous blood into the stent-graft and subsequent thrombosis. Secondly, the positioning of the stent-graft inline with venous flow also may have limited reflux. Current stent-grafts are not designed for lymphatic use and optimal device design could prevent fibrin sheath formation in the future. Addition of an anti-coagulation regimen (similar to routine care after arterial and venous stent placement) could help also prevent occlusion in the future.

In conclusion, bypassing the relative obstruction at the lymphovenous junction by placement of a stent-graft may decompress the thoracic duct and be a promising treatment option for patients in whom TIPS is not feasible or contra-indicated. Further investigation is warranted. 


\section{CONFLICT OF INTEREST AND \\ DISCLOSURE}

HM has received research materials from Guerbet, Inc. The authors declare no competing financial interests exist.

\section{REFERENCES}

1. Witte, MH, AE Dumont, WR Cole, CL Witte, K Kintner: Lymph circulation in hepatic cirrhosis: Effect of portacaval shunt. Ann Intern Med. 70 (1969), 303-310.

2. Dumont, AE, JH Mulholland: Alterations in thoracic duct lymph flow in hepatic cirrhosis: Significance in portal hypertension. Ann Surg. 156 (1962), 668-675.

3. Koep, LJ, R Weil, TE Starz: The technique of prolonged thoracic duct drainage in transplantation. Surg Gynecol Obstet. 151 (1980), 61-64.

4. Ratnayake, CBB, ABJ Escott, ARJ Phillips, JA Windsor: The anatomy and physiology of the terminal thoracic duct and ostial valve in health and disease: Potential implications for intervention. J Anat. 233 (2018), 1-14.

5. Seeger, M, B Bewig, R Günther, et al: Terminal part of thoracic duct: highresolution US imaging. Radiology 252 (2009), 897-904.

6. Sakai, T, S Yabuki, K Chang,et al: Effect of increased systemic venous pressure on thoracic duct and peripheral lymph flow in dogs. Lymphology 18 (1985), 64-67.
7. Allen, SJ, RE Drake, GA Laine, JC Gabel: Effect of thoracic duct drainage on hydrostatic pulmonary edema and pleural effusion in sheep. J. Appl. Physiol. (1985), 71 (1991), 314-316.

8. Donini, I, F Bresadola: Cervical lymph-venous shunt in experimental ascites and in patients with hepatic cirrhosis. Lymphology 7 (1974), 105-108.

9. McGregor, H, G Woodhead, C Hennemeyer, C Harms, M Patel: Stent-graft reconstruction of the lymphovenous junction after complete transection of the cervical thoracic duct. J Vasc. Interv. Radiol. 2020 Sep 16:S1051-0443(20) 30164-0.

10. Chick, JFB, SN Reddy, DA Murrey, et al: Single-session endolymphatic thoracic duct stent-graft placement for recurrent idiopathic chylothorax. J. Vasc. Interv. Radiol. 28 (2017), 1063-1067.

\author{
Hugh McGregor, MD \\ Department of Medical Imaging \\ 1501 N. Campbell Avenue \\ Tucson, AZ, 85724 USA \\ Cell phone: 650-799 7774 \\ Fax: 520-626 7093 \\ E-mail: hughmegregor@radiology.arizona.edu
}

